\title{
The Potential of Nanomaterials for Drug Delivery, Cell Tracking, and Regenerative Medicine 2014
}

\author{
Krasimir Vasilev, ${ }^{1,2}$ Haifeng Chen, ${ }^{3}$ Patricia Murray, ${ }^{4}$ and Diego Mantovani ${ }^{5}$ \\ ${ }^{1}$ Mawson Institute, University of South Australia, Mawson Lakes, SA 5095, Australia \\ ${ }^{2}$ School of Advanced Manufacturing, University of South Australia, Mawson Lakes, SA 5095, Australia \\ ${ }^{3}$ Department of Biomedical Engineering, College of Engineering, Peking University, Beijing 100871, China \\ ${ }^{4}$ Institute of Translational Medicine, The University of Liverpool, Liverpool L69 3GE, UK \\ ${ }^{5}$ Department of Min-Met-Materials Engineering \& CHU de Quebec Research Center, Laval University, Quebec City, Canada
}

Correspondence should be addressed to Krasimir Vasilev; krasimir.vasilev@unisa.edu.au

Received 5 May 2015; Accepted 5 May 2015

Copyright (C) 2015 Krasimir Vasilev et al. This is an open access article distributed under the Creative Commons Attribution License, which permits unrestricted use, distribution, and reproduction in any medium, provided the original work is properly cited.

Nanomaterials have become the building blocks of revolutionary technologies that have opened unprecedented opportunities across the entire global economy. Nanomaterials are particulates of various shapes and forms and assemblies that typically have a size range between 1 and $100 \mathrm{~nm}$. Nature has designed and used nanomaterials for billions of years. For instance, proteins and viruses are complex nanoengineered structures that have been designed by Nature to perform highly specific and refined roles. It was only in the last two decades that we learned how to engineer and use materials at the nanoscale in a relatively large scale. Despite revolutionizing many technologies, these materials are far from the perfection that Nature has created. Thus, scientists and engineers are presented with enormous challenges and opportunities to explore, interrogate, and utilize the unique properties of nanomaterials to improve standards of living and drive economic prosperity.

An area where nanomaterials had a profound implication is medicine. Medical treatments and diagnoses of previously unknown and incurable diseases have become possible. Biological phenomena responsible for various medical conditions have been elucidated through nanotechnology and nanomaterials. For example, magnetic and gadolinium oxide nanoparticles have brought the sensitivity of MRI imaging, magnetic immunoassays, and magnetic hyperthermia to a new dimension. Nanocarriers made possible delivery of pharmaceuticals directly and specifically to cancer cells. Gold nanoparticles have been utilized in thermal imaging and thermal treatment of cancers. Silver nanoparticles have become a common antibacterial agent and were commercially employed on wound dressings and catheters. These are just a few examples of the wealth of revolutionary biomedical applications that have become possible owing to the unique properties of nanomaterials.

This special issue contains a collection of excellent contributions from established laboratories around the world.

In an excellent review paper, C. T. Turner et al. critically review the therapeutic potential of inorganic nanoparticles for the delivery of monoclonal antibodies. Monoclonal antibodies (mAbs) are emerging as the fastest growing area of therapeutic drug development because of their ability to bind with a high degree of specificity to target proteins involved in diseases. However, optimising the delivery, absorption, and distribution of the mAbs, whilst minimising potential side-effects, remained the key problems. This review outlines recent advances in nanoparticle technology and how they can be of benefit as therapeutic mAb delivery/targeting vehicles.

In another review article, R. Yadollahi et al. provide a comprehensive summary of recent developments in nanosuspension technologies for delivery of poorly soluble drugs and their applications in various fields. The problem with many drugs is their poor solubility in aqueous medium and the associated poor bioavailability. Many anticancer and anti-inflammatory drugs fall in this category. To deal with this hurdle in drug therapies, scientists have developed various nanosuspension technologies which have overcome the problem of poor drug aqueous solubility. In the case of nanosuspension, bringing drug particle size to the nanoscale 
allows for an increased surface to volume ratio and with this, improved solubility.

X. Wang et al. report on the "Synthesis of Thermal Polymerizable Alginate-GMA Hydrogel for Cell Encapsulation". These promising three-dimensional porous structures that were evaluated in vivo and in vitro showed potential in in situ tissue engineering scaffold applications. In another report, J. M. Tan et al. studied the sustained release and cytotoxicity of a carbon nanotube-mediated drug delivery system for betulinic acid (BA). These workers used carboxylic acid-functionalized single-walled carbon nanotubes (f-SWCNTs) to bind the water-insoluble anticancer drug BA to produce f-SWCNTs-BA conjugates via noncovalent $\pi-\pi$ stacking interaction. These conjugates were characterized for drug loading capacity, physicochemical properties, surface morphology, releasing characteristics, and cytotoxicity. In another excellent research article, M. Xu et al. reported on the "Enhanced Critical Size Defect Repair in Rabbit Mandible by Electrospun Gelatin/ $\beta$-TCP Composite Nanofibrous Membranes". This work demonstrates that nanofibrous gelatin $/ \beta$ TCP composite membranes could serve as efficient barrier membranes for guided tissue regeneration.

Finally, the Editorial team would like to thank all contributing authors for making this special issue a success.

\section{Acknowledgments}

The Editorial team would like to thank all authors for their excellent contributions. This special issue would not have been of such quality without the constructive criticism of the reviewers. We gratefully acknowledge all colleagues who contributed to the peer review process. Krasimir Vasilev would also like to thank the Australian Research Council for fellowship FT100100292 which allowed him the time to edit this special issue. Haifeng Chen thanks the support from the Ministry of Science and Technology of China (Grants 2012CB933903). Patricia Murray acknowledges support from the UK Engineering and Physical Sciences Research Council (EP/H046143/1).

Krasimir Vasilev Haifeng Chen

Patricia Murray

Diego Mantovani 

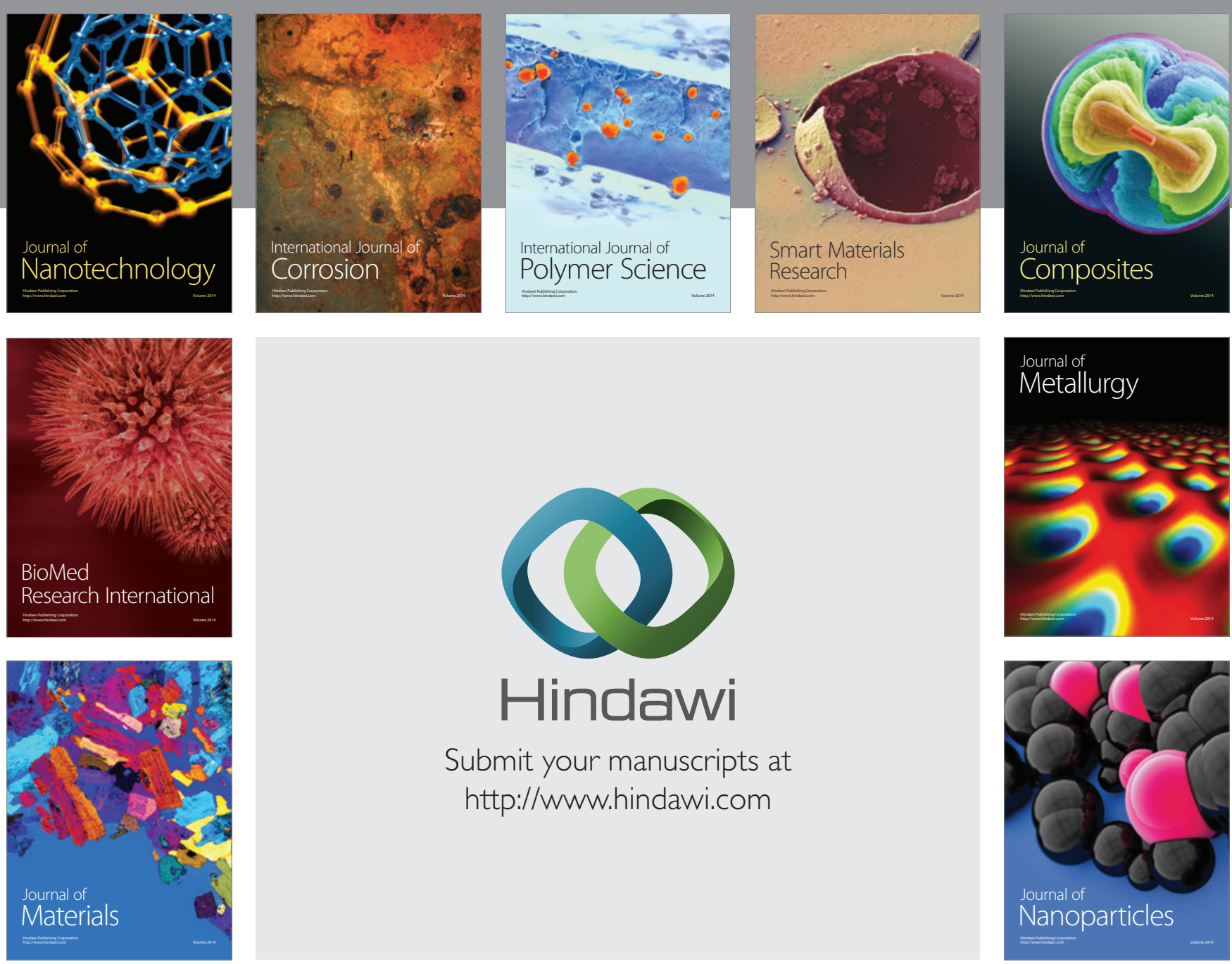

Submit your manuscripts at http://www.hindawi.com
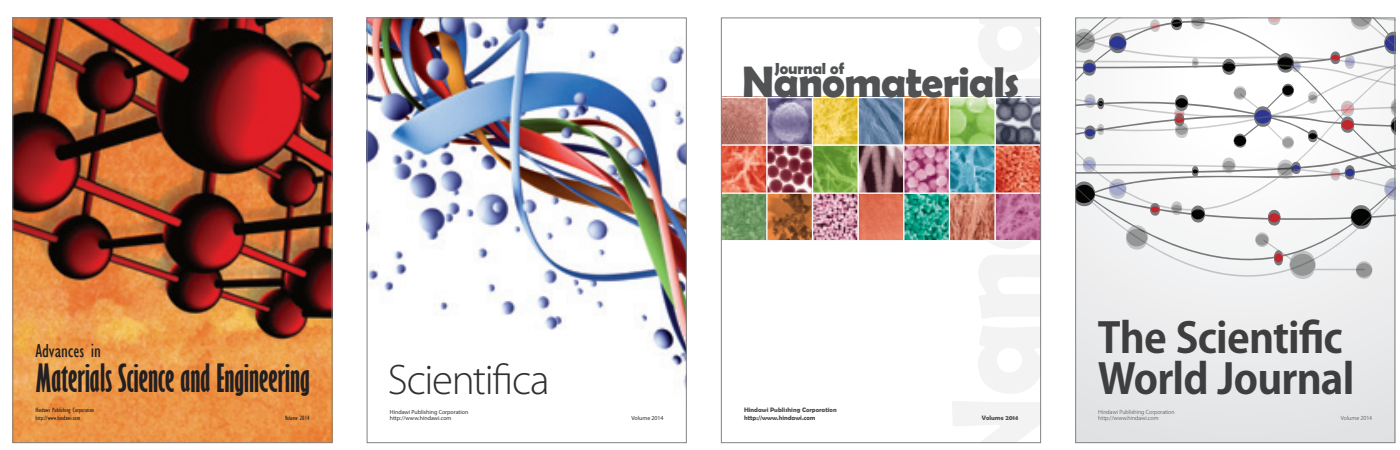

\section{The Scientific World Journal}
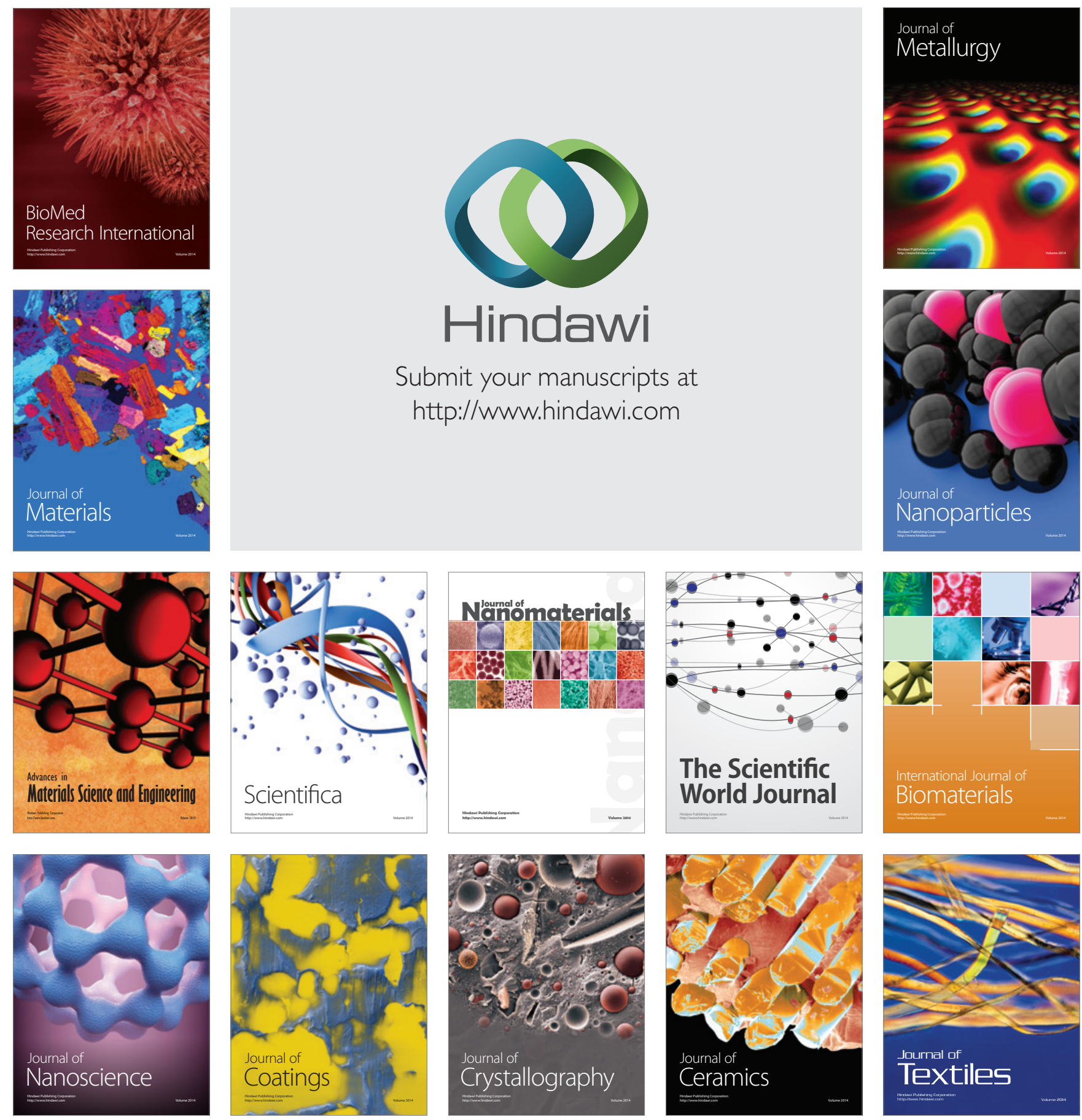